\title{
Relative scales of the GUT and twin sectors in an F-theory model
}

\section{Herbert Clemens ${ }^{a}$ and Stuart Raby ${ }^{b}$}

${ }^{a}$ Mathematics Department, Ohio State University, Columbus OH, U.S.A.

${ }^{b}$ Physics Department, Ohio State University, Columbus OH, U.S.A.

E-mail: clemens.43@osu.edu, raby.1@osu.edu

ABSTRACT: In this note we analyze the relative scales for the GUT and twin sectors in the F-theory model discussed in ref. [1]. There are a number of volume moduli in the model. The volume of the GUT surface in the visible sector (1) (with the Wilson line GUT breaking) defines the GUT scale $M_{G} \sim 2 \times 10^{16} \mathrm{GeV}$ as the unification scale with precise gauge coupling unification of $\mathrm{SU}(3) \times \mathrm{SU}(2) \times \mathrm{U}(1)_{Y}$. We choose the GUT coupling constant, $\alpha_{G}^{-1} \sim 24$. We are then free to choose the ratio $\alpha_{G}(2) / \alpha_{G}(1)=m_{1} / m_{2}$ with $m_{1}$ and $m_{2}$ independent volume moduli associated with the directions perpendicular to the two asymptotic GUT surfaces. We then analyze the effective field theory of the twin sector (2), which may lead to a SUSY breaking gaugino condensate. Of course, all these results are subject to the self-consistent stabilization of the moduli.

KEYwords: Differential and Algebraic Geometry, F-Theory, Supersymmetric Effective Theories, Supersymmetry and Duality

ARXIV EPRINT: 2001.10047 


\section{Contents}

1 Relative scales in F-theory GUT 1

1.1 Semi-stable degeneration of the $F$-theory model 1

1.2 Asymptotic position of $S_{\mathrm{GUT}} \quad 2$

2 Scaling the effective 4-D theory 3

3 Conclusion 5

A Asymptotic position of $S_{\mathrm{GUT}}$ with regards to the Heterotic theory 5

\section{Relative scales in F-theory GUT}

The effective low energy field theory of an $F$-theory GUT is defined on a real 10-dimensional manifold $\mathbb{M}^{10}=\mathbb{R}^{3,1} \times B_{3}$ where $B_{3}$ is a smooth complex projective complex 3-fold with ample anti-canonical bundle, that is, a Fano threefold. Gravity fills all of this real 10dimensional space-time while the GUT theory resides on a smooth two-dimensional anticanonical complex surface $S_{\mathrm{GUT}} \subseteq B_{3}$. The GUT surface $S_{\mathrm{GUT}}$ is defined by the vanishing of $z \in H^{0}\left(K_{B_{3}}^{-1}\right)$.

\subsection{Semi-stable degeneration of the $F$-theory model}

More precisely, as in [1] we consider the family

$$
B_{3, \delta}=\mathbb{P}_{\left[u_{0}, v_{0}\right]} \times B_{2} .
$$

where $B_{3}=B_{3,1}$. For the affine coordinates

$$
(a, b) \in \mathbb{C}^{2} \subseteq \mathbb{P}_{a}^{1} \times \mathbb{P}_{b}^{1}
$$

we set

$$
\begin{aligned}
& a=\delta^{1 / 2} \frac{u_{0}-v_{0}}{u_{0}+v_{0}} \\
& b=\delta^{1 / 2} \frac{u_{0}+v_{0}}{u_{0}-v_{0}}
\end{aligned}
$$

and consider the closure $\mathbb{P}_{[\delta]}$ of the subset

$$
a \cdot b=\delta \in[0,1]
$$

so that

$$
B_{3, \delta}=\mathbb{P}_{[\delta]} \times B_{2} .
$$


Here $B_{2}$ is constructed from the 'last' toric del Pezzo $D_{6}$ endowed with its canonical toric metric $g_{(6)}$ as in (1.3) of [2] with respect to which the $\mathbb{Z}_{4}$-action in [1] is isometric with finite fixpoint set. ${ }^{1}$ We then blow up a single general $\mathbb{Z}_{4}$-orbit, the resulting del Pezzo

$$
D_{2} \subseteq D_{6} \times \mathbb{P}^{1}
$$

where $\mathbb{Z}_{4}$ acts trivially on $\mathbb{P}^{1}$ and the metric $g_{(2)}$ on $D_{2}=B_{2}$ is induced by the above inclusion. Thus we have one real degree of freedom in the choice of the scaling constant on the standard SU (2)-invariant metric on $\mathbb{P}^{1}$.

Similarly $\mathbb{P}_{a}^{1}$ carries the standard SU (2)-invariant metric $g_{a}$ with volume $m_{1}$ and $\mathbb{P}_{b}^{1}$ carries the standard SU (2)-invariant metric $g_{b}$ with volume $m_{2}$. The metric $g_{\delta}$ on $B_{3, \delta}$ is the one induced by restriction of the metric

$$
g_{a} \oplus g_{b} \oplus g_{(2)}
$$

on $\mathbb{P}_{a}^{1} \times \mathbb{P}_{b}^{1} \times B_{2}$. This allows two additional scaling constants, the first giving volume $m_{1}$ to the standard $\mathrm{SU}(2)$-invariant metric on $\mathbb{P}_{a}$ and the second giving volume $m_{2}$ to the standard SU (2)-invariant metric on $\mathbb{P}_{b}$.

The Einstein-Hilbert action is given by

$$
S_{\mathrm{EH}} \sim M_{*}^{8} \int_{\mathbb{R}^{3,1} \times B_{3}} R \sqrt{-g_{\delta}} d^{10} x .
$$

As a consequence, the four-dimensional Planck constant is given by

$$
M_{\mathrm{Pl}}^{2} \simeq M_{*}^{8} \cdot \operatorname{Vol}\left(B_{3, \delta}\right) .
$$

The semi-stable limit of the $F$-theory geometry as $\delta$ goes to zero is the union of two components or 'gauge sectors'

$$
B_{3}^{(1)} \cup B_{3}^{(2)}
$$

crossing along a copy of $B_{2}$ over $(a, b)=(0,0)$ where

$$
\begin{aligned}
& B_{3}^{(1)}=\mathbb{P}_{a}^{1} \times B_{2} \\
& B_{3}^{(2)}=\mathbb{P}_{b}^{1} \times B_{2} .
\end{aligned}
$$

We call $B_{3}^{(1)}$ with induced metric $g_{1}$ the 'visible sector' and $B_{3}^{(2)}$ with induced metric $g_{2}$ the 'hidden or twin sector.' Thus

$$
\operatorname{Vol}\left(B_{3,0}\right)=\operatorname{Vol}\left(B_{2}\right) \cdot\left(m_{1}+m_{2}\right) .
$$

\section{$1.2 \quad$ Asymptotic position of $S_{\mathrm{GUT}}$}

As $\delta$ varies, the GUT surface $S_{\mathrm{GUT}, \delta}$ is defined by the vanishing of

$$
z_{\delta}=\delta \cdot z+(1-\delta) \cdot\left(u_{0}^{2}-v_{0}^{2}\right) \cdot q \in H^{0}\left(K_{B_{3, \delta}}^{-1}\right)
$$

\footnotetext{
${ }^{1}$ This metric is not the Kähler-Einstein metric on $D_{6}$. See [3].
} 
where $q \in H^{0}\left(K_{B_{2}}^{-1}\right)$ is a section in the $(-1)$-eigenspace for the $\mathbb{Z}_{4}$-action on $B_{2}$. We let $C \subseteq B_{2}$ denote the smooth genus-one curve defined by the vanishing of $q$.

Here the gauge action is given by

$$
\left.S_{\text {gauge }} \sim-M_{*}^{6} \int_{\mathbb{R}^{3,1} \times B_{3,0}}\left(\operatorname{Tr}\left(F_{1}^{2}\right) \sqrt{-g_{1}}+\operatorname{Tr}\left(F_{2}^{2}\right) \sqrt{-g_{2}}\right)\right) \delta^{2}\left(z_{0}\right) d^{10} x .
$$

$F_{i}$ denotes the (limiting) curvature tensor of the Yang-Mills connection on the $i$-th gauge sector $B_{3}^{(i)}$. Also $S_{\mathrm{GUT}, 0} \supseteq S_{1} \cup S_{2}$ where

$$
\begin{aligned}
& S_{1}:=\left(\{a=\infty\} \times B_{2}\right) \cup\left(\mathbb{P}_{a}^{1} \times C\right) \\
& S_{2}:=\left(\{b=\infty\} \times B_{2}\right) \cup\left(\mathbb{P}_{b}^{1} \times C\right) .
\end{aligned}
$$

Therefore

$$
\operatorname{Vol}\left(S_{i}\right)=M_{G}(i)^{-4}=\operatorname{Vol}\left(B_{2}\right)+m_{i} \cdot \operatorname{Vol}(C) .
$$

(See appendix A for more detail on the relationship between $S_{1} \cup S_{2}$ and $S_{\mathrm{GUT}, 0}$.)

\section{Scaling the effective $4-\mathrm{D}$ theory}

Hence in the effective 4-dimensional theory we should have GUT coupling constant

$$
\alpha_{G}(i)^{-1} \sim M_{*}^{6} \operatorname{Vol}\left(S_{i}\right) R_{\perp i}^{2}, \quad i=1,2
$$

where $R_{\perp i}^{2} \equiv m_{i}$ is the size of the perpendicular scale for the gauge sectors 1,2 . This calculation is complicated by the fact that each $S_{i}$ has two components whose perpendicular scales do not coincide geometrically nor numerically. The perpendicular scale to the component $S_{i} \subseteq B_{3}^{(i)}$ that projects onto $B_{2}$ is clearly $m_{i}$. However the perpendicular scale for the $\mathbb{P}^{1} \times C \subseteq B_{i}$ must be defined. We do that by recalling that the defining section $q$ of the curve $C \subseteq B_{2}$ is a section of the anti-canonical bundle of $B_{2}$ and this bundle has a metric induced from the fixed toric metric on $B_{2}$. Thus we define the perpendicular scale for the $\mathbb{P}^{1} \times C$ component to be norm

$$
\int_{B_{2}}|q|^{2}
$$

Substituting we obtain

$$
\alpha_{G}(i)^{-1} \sim M_{*}^{6}\left(\operatorname{Vol}\left(B_{2}\right) \cdot m_{i}+\operatorname{Vol}(C) \cdot m_{i} \cdot \int_{B_{2}}|q|^{2}\right), \quad i=1,2
$$

We then find

$$
\begin{aligned}
\alpha_{G}(i) M_{\mathrm{Pl}}^{2} & \sim M_{*}^{2} \frac{\operatorname{Vol}\left(B_{2}\right)\left(m_{1}+m_{2}\right)}{\operatorname{Vol}\left(B_{2}\right) \cdot m_{i}+\operatorname{Vol}(C) \cdot m_{i} \cdot \int_{B_{2}}|q|^{2}} \\
& =M_{*}^{2}\left(\frac{m_{1}+m_{2}}{m_{i}}\right) \frac{\operatorname{Vol}\left(B_{2}\right)}{\operatorname{Vol}\left(B_{2}\right)+\operatorname{Vol}(C) \cdot \int_{B_{2}}|q|^{2}} .
\end{aligned}
$$


Therefore the relative size of the GUT coupling constants and the GUT scales for the visible and twin sectors depends on the relative sizes of the perpendicular directions in $B_{3}$ to the GUT surface.

Let's define the sector labeled (1) as the visible sector with GUT coupling constant, $\alpha_{G}(1)^{-1}=24$ at the GUT scale $M_{G}(1)=2 \times 10^{16} \mathrm{GeV}$. Then the twin sector is sector (2). The ratio $\alpha_{G}(2) / \alpha_{G}(1)=m_{1} / m_{2}$ with $M_{G}(2)>M_{G}(1)$. Let's take $M_{G}(2)=3 \times 10^{16} \mathrm{GeV}$ and $\alpha_{G}(2)^{-1}=8.7$ or $m_{1} / m_{2}=2.8$. Below the scale $M_{G}(2)$ the effective field theory is $\mathrm{SU}(3) \times \mathrm{SU}(2) \times \mathrm{U}(1)_{Y}$, just as in the visible sector. However the twin QCD coupling will become strong at a scale much greater than the visible QCD scale. The effective twin QCD theory has $N_{C}=3$ and $N_{\text {flavors }}=6$. Hence it is described by Seiberg duality [6]. In the magnetic phase, we have an effective superpotential given by

$$
W=q^{i a} T_{i, a}^{j, b} \bar{q}_{j, b}+\lambda_{i j}^{u} q^{i a} H_{u a} \bar{q}_{j 1}+\lambda_{i j}^{d} q^{i a} H_{d a} \bar{q}_{j 2}
$$

where $q(\bar{q})$ are left-handed color triplets (anti-triplets) with the family index, $i=1,2,3$, and $\mathrm{SU}(2)_{\text {isospin }}$ index, $a=1,2$. When $\langle q\rangle_{0}=\langle\bar{q}\rangle_{0}=0$, the theory has a flat direction for the fundamental meson field, $T_{i, a}{ }^{j, b}$. Note, since the twin electroweak group is gauged, we should identify $T_{i, a}{ }^{j, 1} \equiv\left(T_{u a}\right)_{i}{ }^{j}$ and $T_{i, a}{ }^{j, 2} \equiv\left(T_{d a}\right)_{i}{ }^{j}$. The twin supersymmetric $\mathrm{SU}(2) \times$ $\mathrm{U}(1)_{Y}$ gauge interactions introduce a quartic potential for $T_{u}, T_{d}, H_{u}, H_{d}$ such that there is a flat direction for $\left\langle\left(T_{u 1}\right)_{i}{ }^{j}\right\rangle=\left\langle\left(T_{d 2}\right)_{i}{ }^{j}\right\rangle=T \delta_{i}{ }^{j}$ and $H_{u 1}=H_{d 2}=T$. Then all twin quarks and leptons obtain mass at the scale $T$ and, moreover, the twin electroweak gauge symmetry is broken down to twin $\mathrm{U}(1)_{E M}$. For $T \sim M_{G}(2)$, we find a twin gluino condensate occurs at the scale $\Lambda_{\mathrm{tQCD}}=T \exp \left(-\frac{2 \pi}{9 \alpha_{G}(2)}\right) \sim 6.9 \times 10^{13} \mathrm{GeV}$.

We expect that the effective $4 \mathrm{D}$ QCD Lagrangian contains a term

$$
L \supset \frac{1}{2} \int d^{2} \theta \sum_{i=1}^{2}\left(\frac{S(i)}{4} \operatorname{Tr} W^{\alpha} W_{\alpha}(i)+\text { h.c. }\right)
$$

with

$$
S(i)=\frac{1}{4 \pi \alpha_{G}(i)}+i \theta=e^{\ln \left(K(i) m_{i}\right)-\phi}+i b,
$$

where $\phi, b$ is the dilaton, axion fields, and $m_{i}$ is as above the volume of the $\mathbb{P}^{1}$ direction perpendicular to the GUT surface in the visible and twin sectors and $K(i) \sim M_{*}^{6} M_{G}(i)^{-4}$. We also expect a non-perturbative superpotential term of the form [7-9]

$$
W_{\mathrm{NP}} \supset A\left[e^{-8 \pi^{2} S(2) / 9} T\right]^{3} .
$$

As a consequence, the twin QCD condensate will contribute SUSY breaking effects to both the twin and visible sectors of the theory. In this local SUSY theory, we find an effective low energy SUSY breaking scale given by

$$
m_{3 / 2}=\Lambda_{\mathrm{tQCD}}^{3} / M_{\mathrm{Pl}}^{2} \sim 60 T e V .
$$

Of course, whether supersymmetry is broken (or not) depends on stabilizing all the moduli.

The low energy supersymmetric theory contains 3 families of twin neutrino superfields (assuming that the three right-handed neutrinos obtain mass near the GUT scale), 
19 chiral charged and neutral Higgs superfields (which include the massless components of $H_{u}, H_{d}$ and $\left.T_{u}, T_{d}\right)$, and the twin photon superfield. Renormalizing from $M_{G}(2)$ we find $\alpha^{t E M}\left(m_{3 / 2}\right) \sim 1 / 105$. There do not appear to be any portals to the twin sector. Clearly, the twin sector introduces new candidates for dark matter, but a complete analysis of the cosmological implications of this sector for the theory is beyond the scope of the present paper.

\section{Conclusion}

In conclusion, we have analyzed the relative scales of the visible and twin sectors in the global $F$-theory GUT with Wilson line breaking given in [1]. We have found that there is sufficient freedom to have independent GUT scales and couplings in order to have interesting physics coming from the twin sector. In particular, if we assume that the GUT coupling for the twin sector is larger than that of the visible sector, then it is possible to spontaneously break the twin electroweak theory at the GUT scale with all twin quarks and charged leptons obtaining mass at that scale. In addition, a twin gluino condensate can then occur at a scale of order $\Lambda_{\mathrm{tQCD}} \sim 6.9 \times 10^{13} \mathrm{GeV}$ which leads to an effective low energy SUSY breaking scale, $m_{3 / 2}=\Lambda_{\mathrm{tQCD}}^{3} / M_{\mathrm{Pl}}^{2} \sim 60 \mathrm{TeV}$ which affects both the twin and visible sectors. There is clearly more analysis that needs to be done on the consequences of these results, including the stabilization of moduli, which we leave for the future. For example, the model also includes $11 D_{3}$ branes and fluxes which must be considered [10].

\section{A Asymptotic position of $S_{\mathrm{GUT}}$ with regards to the Heterotic theory}

The Heterotic dual is the smooth Calabi-Yau threefold $\left[V_{3}=B_{3}^{(1)} \cap B_{3}^{(2)}\right]$ over $a=b=$ 0 . Each $B_{3}^{(i)}$ encodes the structure of an $E_{8}$-bundle with Yang-Mills connection on the Heterotic model $V_{3}$ via the equivalence of Yang-Mills $E_{8}$-bundles and $d P_{9}$-bundles given by the dictionary in $\S 4.2$ of [4]. A slightly subtle point in the encoding is that the factor $q$ in $z_{0}$ is irrelevant to the determination of the $E_{8}$-bundle, since over each point $b_{2} \in B_{2}$, $q\left(b_{2}\right)$ simply re-scales the vector $[s, t]$ in the Weierstrass form

$$
\left[y^{2}=4 x^{3}-\left(g_{2} t^{4}-\beta_{1} s t^{3}-\ldots-\beta_{4} s^{4}\right) x-\left(g_{3} t^{6}-\alpha_{2} s^{2} t^{4}-\ldots-\alpha_{6} s^{6}\right)\right]
$$

for the equation of each $d P_{9}$-fiber of $B_{3}^{(i)} / B_{2}$. Thus the Weierstrass form, and so the isomorphism class of the $d P_{9}$-bundle, is unaffected. Said otherwise, Heterotic " $S_{\mathrm{GUT}}$ " is simply the union of the section $\{a=\infty\}$ of $B_{3}^{(1)} / B_{2}$ and the section $\{b=\infty\}$ of $B_{3}^{(2)} / B_{2}$.

Open Access. This article is distributed under the terms of the Creative Commons Attribution License (CC-BY 4.0), which permits any use, distribution and reproduction in any medium, provided the original author(s) and source are credited.

\section{References}

[1] H. Clemens and S. Raby, Heterotic-F-theory duality with Wilson line symmetry-breaking, JHEP 12 (2019) 016 [arXiv: 1908.01913] [INSPIRE]. 
[2] V. Guillemin, Kähler structures on toric varieties, J. Diff. Geom. 40 (1994) 285.

[3] C. Doran et al., Numerical Kähler-Einstein metric on the third del Pezzo, Commun. Math. Phys. 282 (2008) 357 [hep-th/0703057] [INSPIRE].

[4] R. Friedman, J. Morgan and E. Witten, Vector bundles and F-theory, Commun. Math. Phys. 187 (1997) 679 [hep-th/9701162] [INSPIRE].

[5] C. Beasley, J.J. Heckman and C. Vafa, GUTs and exceptional branes in F-theory - II: experimental predictions, JHEP 01 (2009) 059 [arXiv:0806.0102] [INSPIRE].

[6] N. Seiberg, Electric-magnetic duality in supersymmetric nonAbelian gauge theories, Nucl. Phys. B 435 (1995) 129 [hep-th/9411149] [INSPIRE].

[7] H.P. Nilles, Gaugino condensation and SUSY breakdown, hep-th/0402022 [INSPIRE].

[8] L. Görlich, S. Kachru, P.K. Tripathy and S.P. Trivedi, Gaugino condensation and nonperturbative superpotentials in flux compactifications, JHEP 12 (2004) 074 [hep-th/0407130] [INSPIRE].

[9] B. Dundee, S. Raby and A. Westphal, Moduli stabilization and SUSY breaking in heterotic orbifold string models, Phys. Rev. D 82 (2010) 126002 [arXiv: 1002.1081] [INSPIRE].

[10] S. Cecotti, M.C.N. Cheng, J.J. Heckman and C. Vafa, Yukawa couplings in F-theory and non-commutative geometry, arXiv:0910.0477 [INSPIRE]. 